\title{
ACTIVE MICRONEEDLES WITH INTEGRATED FUNCTIONALITY
}

\author{
John Brazzle, Dan Bartholomeusz, Rupert Davies, and Joseph Andrade \\ ${ }^{1}$ Department of Bioengineering, University of Utah \\ Salt Lake City, Utah 84112 \\ Richard A. Van Wagenen ${ }^{1,2}$ \\ ${ }^{2}$ Protein Solutions \\ Salt Lake City, Utah 84112
}

\begin{abstract}
The focus of this work is the design, fabrication, and characterization of a new class of biomedical micro systems, the active microneedle. Active microneedle systems represent an advancement over current microneedle technologies through the integration of additional functionality (e.g. biochemical sensing, mechanical, etc.). The active microneedles described in this paper include the following additional functionality: integration of multiple inlet/output ports, multiple lumens (flow channels), and bioluminescence based biosensors for monitoring metabolic levels (e.g. creatine and glucose).
\end{abstract}

\section{INTRODUCTION}

Both hollow and solid microneedles and microneedle arrays have been demonstrated by a number of research groups over the past few years. Initially, only microneedles made of solid silicon had been realized [1]. Najafi and Wise developed these early microneedles that were used as neural microprobes. Recently, solid silicon microneedles fabricated by Henry et. al. were formed using reactive ion etching [2]. The resulting planar needle array consisted of $20 \times 20$ needles for increasing the skin permeability to drugs. Lin, Pisano, and Muller were first to present hollow silicon processed microneedles $[3,4]$. These microneedles were fabricated on silicon substrate and used silicon nitride to fully enclose the channels. Later, Chen and Wise [5] presented hollow silicon microneedles for neural recording and drug delivery. These two approaches utilize a sacrificial processing technique that consumes the substrate on the devices are built. In contrast, Talbot and Pisano [6] have incorporated a polysilicon micromolding process to develop hollow microneedles. These hollow microneedles are fabricated by micromolding polysilicon (polymolding) in silicon micromolds.

Previously, we reported micromachined needles and needle arrays of hollow metallic needles for minimally invasive drug delivery and biofluid extraction $[7,8]$. These microneedles have the capability to deliver drugs subcutaneously while while minimizing the pain inflicted to patients. Recent advancements in

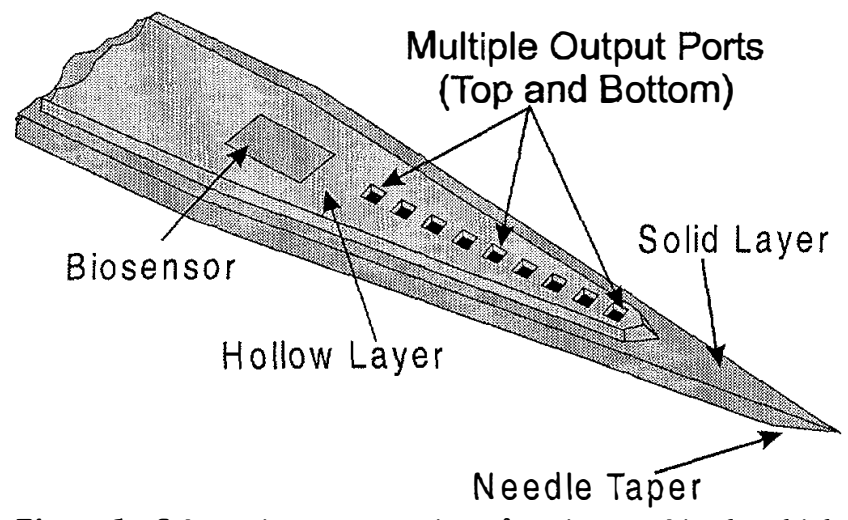

Figure 1. Schematic representation of a micromachined multiple output port needle with an integrated biosensor. the design and fabrication of hollow metallic microneedles has led to improved fluid handling capabilities, as well as, increased functionality. Recently, McAllister et al. [9] have reported hollow $\mathrm{NiFe}$ microneedle arrays.

The paper presents the design, fabrication, and characterization of a hollow metallic active microneedle. Figure 1 is a schematic representation of the described microneedle with an integrated biosensor. The active microneedle includes design features such as tapered needle tips; multiple output ports on the back and front of each needle; multiple lumens and multiple input ports, Figure 2, and bioluminescence based biosensors for monitoring metabolic levels.

\section{FABRICATION AND PACKAGING}

The micromachined multiple output port needles are fabricated using extensions of previously reported micromachining technologies $[10,11,12]$. The fabrication process is low temperature and is compatible with integrated circuit (IC) technology as a post process. Initially, a metal system of adhesion layers and an electroplating seed layer are electron beam evaporated onto a silicon wafer. Using standard thick photoresist micromolding techniques, this metal layer is patterned and $20 \mu \mathrm{m}$ of palladium is electroplated to form the solid layer or bottom wall of the microneedle [13]. The use of palladium as a structural material provides high mechanical strength and durability, as well as, biocompatibility for use in biomedical applications $[14,15,16]$. Next, $20 \mu \mathrm{m}$ of commercially available thick photoresist, AZ4620, is deposited and patterned into sacrificial structures. These sacrificial structures serve to precisely define the inner dimension of each microneedle. $800 \AA$ of gold is sputter deposited onto the sacrificial photoresist structures to act as an electroplating seed layer for top shell electroplating. Next, a $20 \mu \mathrm{m}$ layer of palladium is electroplated to form the top and side walls (hollow layer) of the microneedle. The sacrificial thick photoresist is then removed

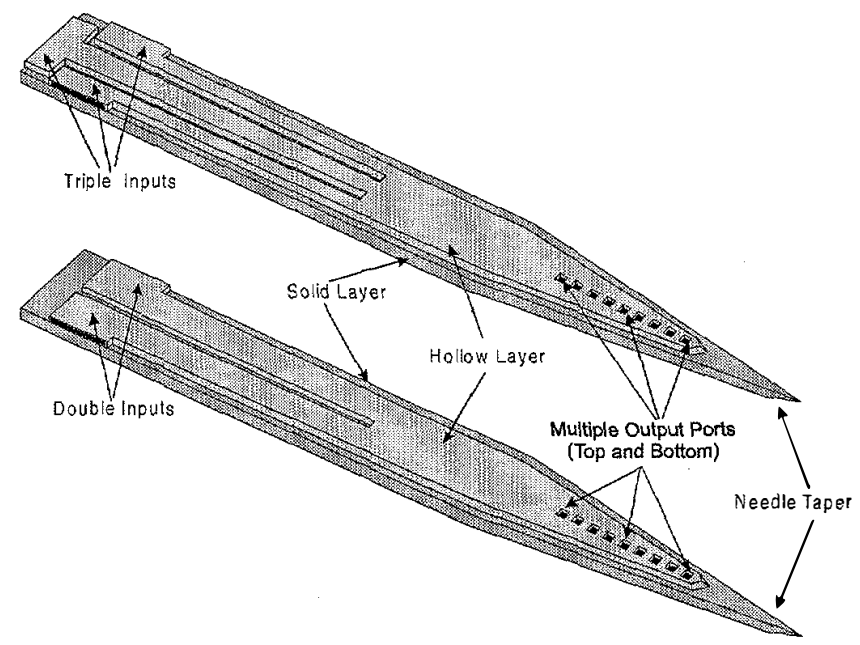

Figure 2. Schematic representation of multi-lumen design for the active microneedles. 
using a sequential rinse in acetone, isopropanol, and de-ionized water, producing the hollow metallic microneedles. In the final step, the microneedles are released from the silicon substrate by first removing the chromium layer to expose the copper seed layer. Then the copper seed layer is etched away from underneath the needles by placing the wafer in a solution of ammonium hydroxide saturated with cupric sulfate. The solid silicon substrate is not consumed in this process and may be re-used. Using the given process offers the flexibility to integrate many additional functions directly onto the microneedle. Additionally, the basic process allows for great latitude in the routing and branching of the fluid

\section{BIOLUMINESCENCE}

Bioluminescence-based analysis is 100 to 1000 times more sensitive than conventional chromogenic (absorbance) measurements and is accurate over a five or more, orders of magnitude concentration range [17]. Firefly bioluminescence occurs by enzyme-catalyzed oxidation of luciferin utilizing adenosine triphosphate (ATP) [18]. Bacteria bioluminescence is closely coupled to nicotinamide adenine dinucleotide hydride (NADH). Since most of biochemistry depends on ATP and/or $\mathrm{NADH}$, nearly all metabolic reactions can be monitored by bioluminescence via one or more enzymes catalyzed and linked reactions $[\mathbf{1 7}, 18]$. During the production or consumption of a metabolite of interest, enzymes linked reactions will cause the production or consumption of ATP (or NADH). Detectable light is then produced via the following reaction (for ATP):

$\mathrm{ATP}+\mathrm{O}_{2}+\mathrm{D}$-Luciferin $+\mathrm{Mg}^{++} \stackrel{\text { Luciferase }}{\longrightarrow}$

Oxyluciferin $+\mathrm{AMP}+\mathrm{CO}_{2}+\mathrm{Ppi}+$ Light $(560 \mathrm{~nm})$

The change in light intensity will be stoichiometrically proportional to the time changing concentration of ATP (and thus proportional to the metabolite of interest). A photodiode or charge-coupled device (CCD) can be used for detection. Depending on the instrument used to detect the luminescence, nanomolar, picomolar, and even femtomolar analyses are possible [17]. With increased sensitivity, smaller amounts of sample fluid are needed for accurate analysis. $0.05-\mu \mathrm{L}$ sample size that can be drawn by micromachined needles can painlessly access small amounts of body fluid that can be analyzed via bioluminescence.

The transmittance of light through a media across length $\lambda$ is defined by Beer's law, $T=10^{-A b s o r b a n c e}$, where Absorbance $=$ $\Gamma_{\lambda} \mathrm{C}_{B}(\lambda-x)$ and $\Gamma_{\lambda}$ is the molar absorption coefficient in $\mathrm{dm}^{3} /$ molEcm and $C_{B}$ is the concentration in mole/dm ${ }^{3}$ [19]. If the light is generated within a homogeneous media, the ransmitting light is integrated along the length it travels and is then defined as:

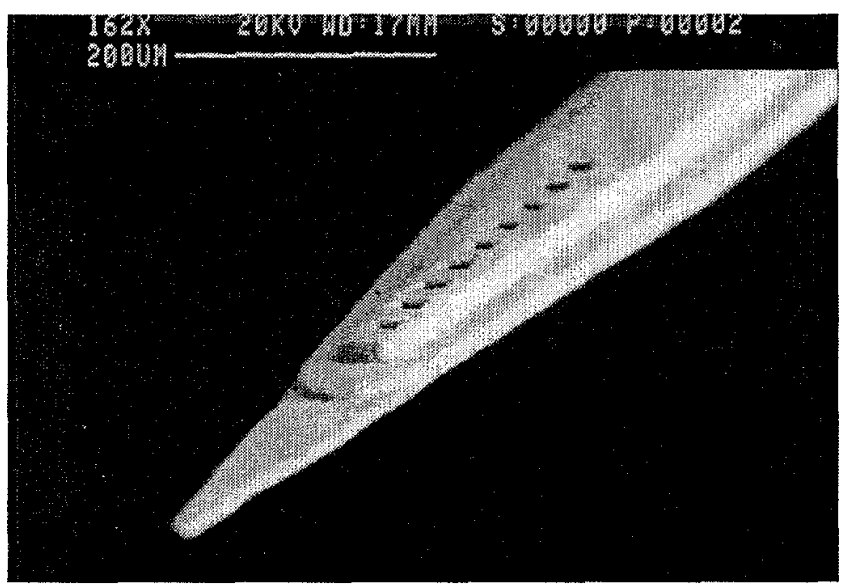

Figure 3. SEM of a multi-lumen, multiple output port micro needle. Shaft dimensions are $200 \mu \mathrm{m}$ wide and $60 \mu \mathrm{m}$ thick. Tip dimensions are less than $15 \mu \mathrm{m} X 15 \mu \mathrm{m}$. Output ports are $30 \mu \mathrm{m}^{2}$.

$$
T_{\text {Total }(\lambda)}=\int_{0}^{\lambda} 10^{-\varepsilon_{\lambda} C_{B}(\lambda-x)} d x=\frac{\left(1-10^{-\lambda}\right)}{\operatorname{Ln} 10} .
$$

where $\Gamma_{\lambda} C_{B}$ is assumed to be 1 for simplicity. For a pyramid (which is the shape of the etched wells in the $\langle 100\rangle \mathrm{Si}$ wafers) with a peak of length $\lambda$, the transmittance is integrated through the viewing area and is equal to $T_{\text {Total }(\lambda)} \mathrm{Ea}^{2} / 3$. The assumption is that only the light being transmitted toward the viewing window is integrated and light traveling away from the viewing window is absorbed by the substrate. (Light a $560 \mathrm{~nm}$ is actually absorbed by crystallized $\mathrm{Si}$ ). Thus, linear correlation of a volume verses integrated luminosity plot, for a given viewing window, should indicate that transmittance is proportional to signal intensity (averaged CCD counts over the viewing area). A flat bottom well with a reflective surface will have the transmittance of $T_{\text {Total }(2 \lambda) \text {, }}$ and will be about 2 time larger than $T_{\text {Total }(\lambda)}$ for $\lambda<<1-\mathrm{cm}(0.005-$ $0.025-\mathrm{cm}$ in this experiment). Thus, the slope of a linear fit of volume verses integrated luminosity plot, for a reflective surface of a given viewing window, should be about 2 times that of the slope for a non-reflective surface due to the increased transmittance.

\section{RESULTS}

\section{Fabrication}

The needle arrays fabricated to date include those electroformed from low-stress nickel sulphamate, gold cyanide, and palladium electroplating solutions $[20,21]$. Individual hollow metallic micromachined needles with multiple output ports fabricated to date have an inner cross-sectional area of $140 \times 21$ $\mu \mathrm{m}^{2}(\mathrm{~W} \times \mathrm{H})$ with outer dimensions of $200 \times 60 \mu \mathrm{m}^{2}$. A micromachined needle fabricated on silicon and released from its substrate is shown in Figure 3. The needle shaft dimensions are $200 \mu \mathrm{m}$ wide and $60 \mu \mathrm{m}$ thick while the tip dimensions are less than $15 \times 15 \mu \mathrm{m}^{2}$. The length of the tapered portion of the needle shaft is $1.0 \mathrm{~mm}$ and the distance from the tip to the first output port is approximately $300 \mu \mathrm{m}$. The total length of the microneedle is 6.0 $\mathrm{mm}$ with input port inner dimensions of $140 \mu \mathrm{m}$ wide and $21 \mu \mathrm{m}$ high. The wall thickness of the needle is approximately $20 \mu \mathrm{m}$ and the microneedle output ports are on the top and bottom with dimensions of $30 \mu \mathrm{m}^{2}$. The output ports are separated by $30 \mu \mathrm{m}$ and there are nine ports on top and 12 ports on the bottom. Bioluminescent detection of the biosensor output is performed at the output ports of the microneedle. Biosensing reagents are drawn into and dried down onto the inner walls of the hollow microneedle.

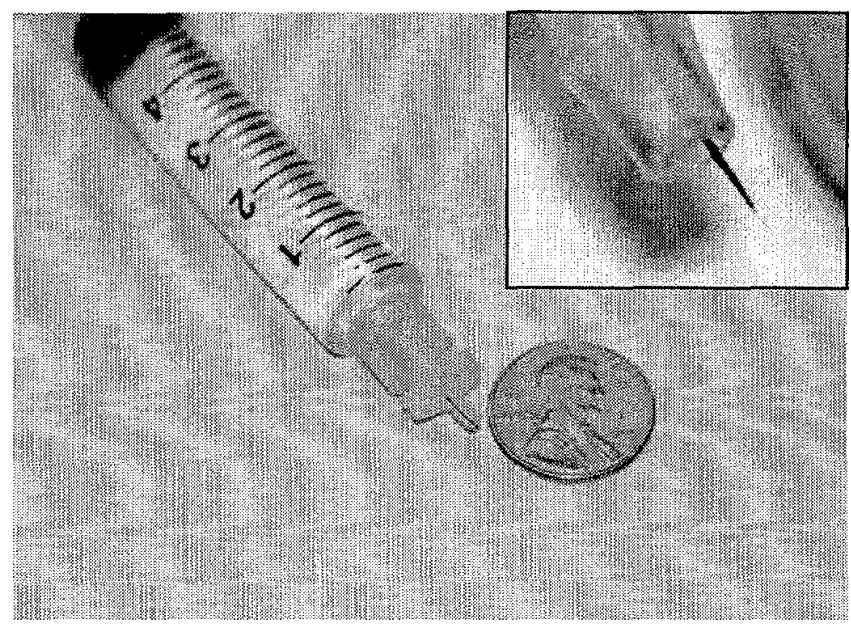

Figure 4. Photograph of packaged microneedle connected to standard 5cc syringe as compared to a penny. Insert: Close-up of packaged microneedle. 
The completed micromachined needle structures were packaged into a standard luer-lock fitting using a polymeric medical-grade UV-curable adhesive (3321, Loctite Corp.). This interface between the microneedle and syringe consists of a simple female luer-to-1/16" barb adapter manufactured by Westem Analytical (part\#p-857x). Figure 4 is a photograph of a packaged microneedle connected to a standard 5cc syringe as compared to a penny. The insert of Figure 4 shows a close-up of the packaged microneedle that has been secured with UV epoxy on the barbed end of the interface. The UV curable epoxy was found to permanently affix the microneedle to the interface while providing a leak resistant seal. Fluid flow experiments were performed on five packaged microneedles at pressures ranging from $1 \mathrm{psi}$ to $70 \mathrm{psi}$ [9].

\section{Bioluminescence Studies}

In order to determine the feasibility and the physical limitations of using bioluminescence as a means for highly sensitive analyte measurement of small sample volumes, $\mu$ reaction chambers ( $\mu \mathrm{RCs}$ ) were fabricated on silicon wafers using $\mathrm{KOH}$ anisotropic wet etching. An ATP firefly luciferase/luciferin solution was placed in the $\mu \mathrm{RCs}$ and observed through a close up lens with a CCD. The integrated CCD signal was recorded and compared with well size and depth. The attenuation of the CCD signal was also observed for wafers coated with titanium (500A) followed by chromium ( $1500 \AA ̊ A)$.

A 5-mL firefly luciferase/luciferin solution consisted of $1.25-\mathrm{mg} / \mathrm{mL}$ bovine serum albumin (Sigma - reconstituted into the solution. Used for coating the glass vial to prevent denaturing of the luciferase), $1.25-\mathrm{mM}$ ethylene diaminetetra acetic acid (Sigma), $12.5-\mathrm{mM} \mathrm{Mg}^{++}\left(\mathrm{Sigma}-\right.$ from $\left.\mathrm{MgSO}_{4}\right), 1.84-\mu \mathrm{M}$ firefly luciferase (Promega), and $1.25-\mathrm{mM}$ luciferin (Biosynth) in a $1.25 \mathrm{mM}$ glycyl-glycine buffer. This mixture was able to maintain $90 \%$ activity for about 20 -hrs when stored in the dark. A $5-\mathrm{mM}$ ATP, glycyl-glycine buffer solution was also prepared. $20-\mu \mathrm{L}$ of the firefly luciferase/luciferin solution was pipetted into $5-\mu \mathrm{L}$ of the ATP solution that resulted in a 1.0-mM ATP mixture, saturated

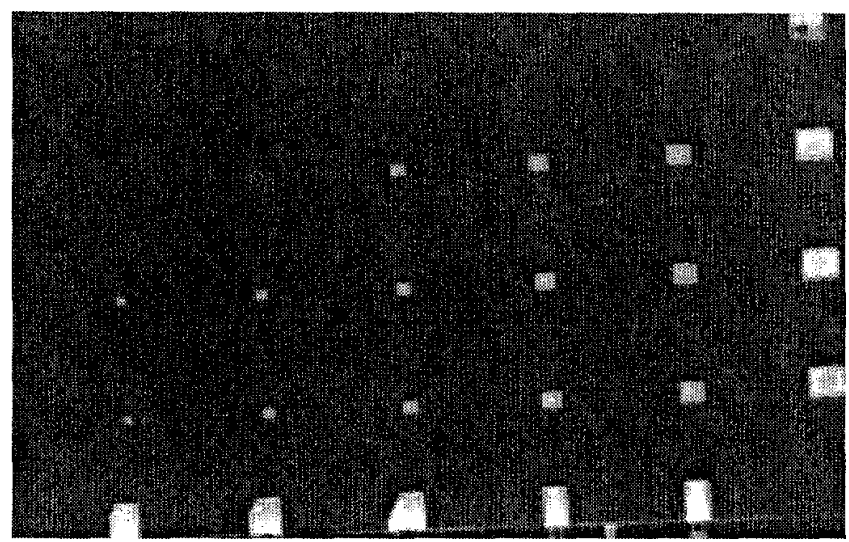

Figure 5. 20-sec integration of bioluminescence for the wafer that was etched 242.1- $\mu \mathrm{m}$ and coated with Ti/Cr. The $\mu R C s$ seen here are the 750, 500, 400,300, 250, and 200- $\mu \mathrm{m}$ wide squares.

\begin{tabular}{|r|l|l|}
$\mu R C$ Width \\
$(\mu \mathrm{m})$ & $\begin{array}{l}\text { P value from T-test between } \\
\text { Intensity/Nolume Slopes of } \\
\text { Non-Reflective } \mu \text { RCs and } \\
\text { Reflective } \mu R C s\end{array}$ & $\begin{array}{l}\text { Ratio of Intensity/Nolume } \\
\text { Slopes for Reflective and } \\
\text { Non-Reflective Substrates }\end{array}$ \\
\hline 750 & 0.00001 & 3.02 \\
500 & 0.002 & 2.37 \\
400 & 0.004 & 4.94 \\
300 & 0.005 & 5.11 \\
250 & 0.36 & 2.87 \\
200 & 0.030 & 1.07 \\
150 & 0.72 & 0.88 \\
100 & 0.55 & 0.21 \\
\hline
\end{tabular}

Table 1. Statistical comparison of the intensity/volume slope relationships for reflective vs. non-reflective surfaces of the same viewing window size.

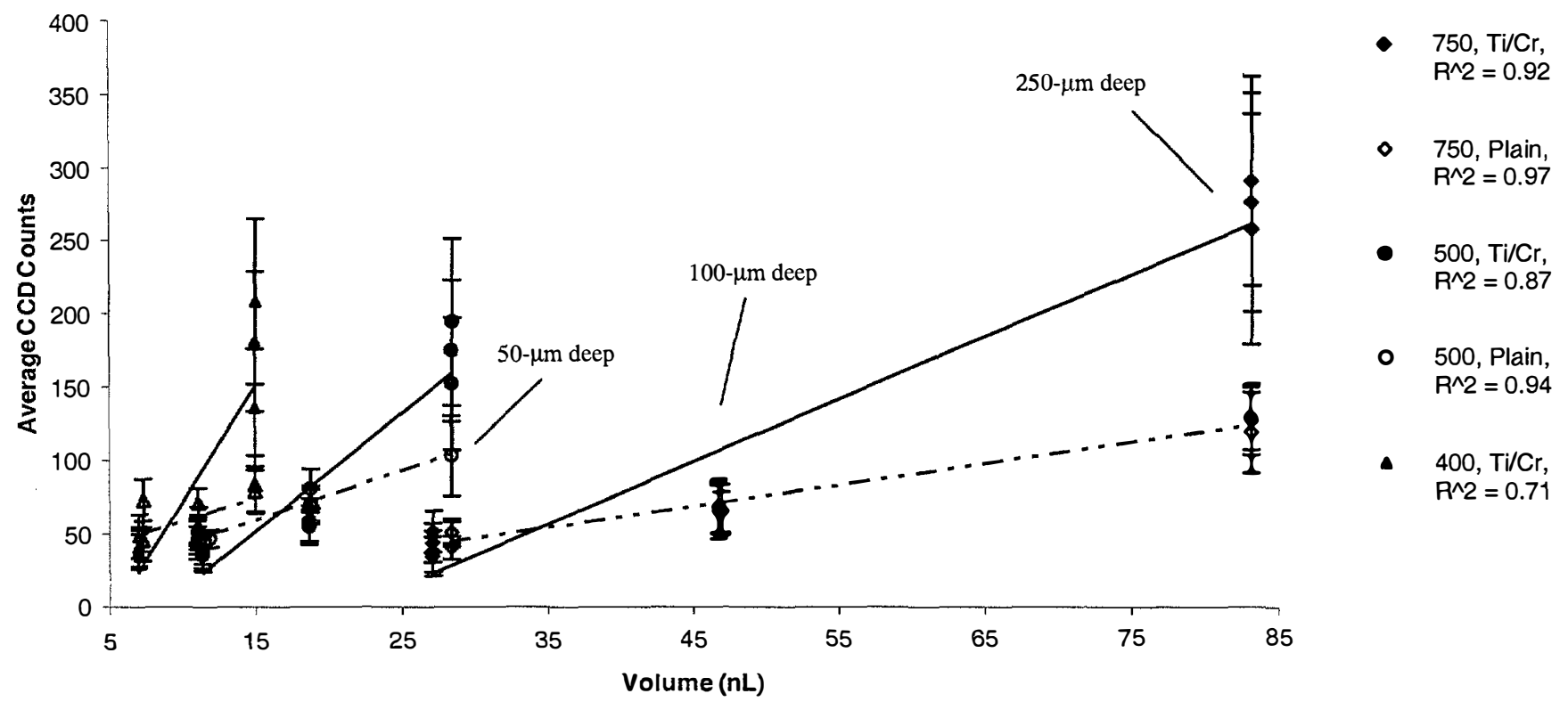

Figure 6. Average CCD counts from the 20-sec integrated CCD reading for different sample volumes. Data was plotted in sets for the same viewing area (or $\mu R C$ square width). Data was also separated according for Ti/Cr reflective substrates and plain, non-reflective, substrates. For

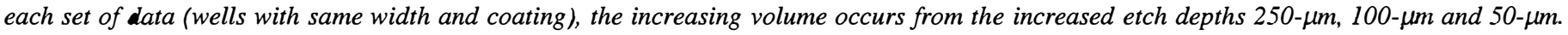
Data from the different etch depths are pointed out for the 750- $\mu m$ wide wells as an example. The higher intensity values occur for the deeper wells. The intensity/volume slope is greater for Ti/Cr reflective substrates than for plain substrates. 
with luciferase and luciferin (which means the reaction rate was at it's peak). After the solution mixed, $20-\mu \mathrm{L}$ of it was pipetted on an area about $20 \times 15-\mathrm{mm}$ wide. A thin glass cover slip was placed on the solution, starting at one end and tilting the cover slip as it was laid down, so that the excess bioluminescent fluid would disperse. For high concentrations of ATP $(>8-\mu \mathrm{M})$, the initial mixing of ATP with the luciferase/luciferin solution causes a peak luminescence within 3 seconds. The luminescence then tapers and levels off after 1 minute. Therefore, the light measurements for this experiment was integrated for 20 seconds, 2 minutes after the ATP and luciferase/luciferin solution were mixed, with the light intensity essentially constant. An ST6-A CCD camera, by Santa Barbara Instruments Group was fitted with an Olympus wide-angle lens and close-up ring. The experimental substrates were focused $55-\mathrm{mm}$ below the lens with the aperture set at 2.8. The field of view was about $20 \times 15-\mathrm{mm}$. The camera was operated at $-20.00^{\circ} \mathrm{C}$. 1.0-minute after the luciferase/luciferin solution was added to the ATP, a 20-sec dark field exposure was taken with the CCD. 2-min 5-sec after the ATP and luciferase/luciferin solution mixed, the CCD integrated a 20 -sec exposure of the bioluminescent reaction while shrouded in a darlroom. The resulting image was saved as a TIF file (Range: $0-400$ ). Scion Image (based on the NIH image software) was used to determine the average and standard deviation of the pixel values for each $\mu \mathrm{RC}$.

Figure 5 shows an image of the 20-second integration of bioluminescence recorded by the CCD camera. One pixel of the CCD image was equal to $46.875-\mu \mathrm{m}$. Actual results failed to show light emitting from the $75,50,25$, and $10-\mu \mathrm{m}$ wide wells on all substrates. Figure 6 shows the average CCD counts from the 20sec integrated CCD reading for different sample volumes. Table 1 statistically compares the intensity/volume slope relationships for reflective vs. non-reflective surfaces of the same viewing window size. The reflective wells with intensity/volume slopes that were statistically different from the intensity/volume slopes for the nonreflective surfaces $(\mathrm{P}<0.05$ using the $\mathrm{T}$-test $)$ were over two times greater. This follows the hypothesis that transmittance is nearly doubled for chromium (reflectivity=0.67@560 nm) coated versus uncoated silicon (reflectivity=0.33@ @ $560 \mathrm{~nm}) \mu \mathrm{RCs}$.

The intensity/volume slope also increases as the $\mu \mathrm{RC}$ square width decreases. This is ideal for using long, narrow microneedles as the $\mu \mathrm{RC}$. Table 2 statistically compares the intensity/volume slopes between reflective wells of different viewing widow sizes. It shows that there is a significance increase in the intensity/volumes slopes, as the $\mu \mathrm{RC}$ width decreases. The increase in intensity/volume is only significant down to wells that are $400-\mu \mathrm{m}$ wide. For smaller $\mu \mathrm{RC}$ widths $(150-300-\mu \mathrm{m})$, the non-reflective substrates show little correlation between average CCD counts and sample volumes $\left(R^{2}<0.25\right)$. The Ti/Cr reflective subsuates still show some correlation $\left(R^{2}>0.25\right.$ for $\mu R C s 250-\mu m$ wide and wider) between intensity and volume. However, as the $\mu \mathrm{RC}$ square width decreases $(250-\mu \mathrm{m}$ wide and smaller), there is little difference in signal intensity values for different etch depths.

\begin{tabular}{|c|c|c|}
\hline $\begin{array}{l}\mu R C \text { Square } \\
\text { Widths Being } \\
\text { Compared }(\mu \mathrm{m})\end{array}$ & $\begin{array}{l}P \text { value from } T \text {-test between } \\
\text { Intensity/Volume Slopes } \\
\mu R C \text { S being compared }\end{array}$ & $\begin{array}{l}\text { Ratio of Intensity/Volume } \\
\text { Slopes for Smaller } \mu \text { RC } \\
\text { Widths Verses Wider } \mu \text { RC } \\
\text { Widths }\end{array}$ \\
\hline $500 / 750$ & 0.004 & 1.89 \\
\hline $400 / 500$ & 0.04 & 1.98 \\
\hline $300 / 400$ & 0.28 & 1.59 \\
\hline $250 / 300$ & 0.89 & 1.11 \\
\hline $200 / 250$ & 0.81 & 1.28 \\
\hline $150 / 200$ & 0.91 & 0.73 \\
\hline
\end{tabular}

Table 2. Statistical comparison of the intensity/volume slopes between reflective wells of different viewing widow sizes. The intensity/volume slope ratio is the slope for the smaller window over the slope for the next larger window.
The error in the signals for wells $200-\mu \mathrm{m}$ wide and smaller is large enough to overlap with the background intensity. This suggests that $250-\mu \mathrm{m}$ that smallest well window that would produce a discernable intensity signal. However, this does not mean that narrower, deeper $\mu \mathrm{RCs}$ like microneedles could not produce a good signal because their longer depths are able to hold a larger sample volume, which would produce a stronger signal.

\section{CONCLUSION}

Both silicon and metal electroformed micro needle technologies have been previously developed. This paper provides a basis for the next technological step in micro needle technologies through the development of active micro needles. Active micro needles include the integration of additional functionality into the basic micro needle structure. Additional functionality such as multiple lumens, multiple input and output ports, and the integration of biosensing capabilities has been shown.

\section{REFERENCES}

1. K. Najafi and K.D. Wise, "An implantable multielectrode array with onchip signal processing," IEEE J. Solid-State Circuits, SC-21, pp. 1035-44, Dec. 1986.

2. S. Henry, D.V. McAllister, M.G. Allen, and M.R. Prausnitz, "Micromachined needles for the transdermal delivery of drugs", in IEEE MEMS Conference, Heidelberg, Germany, Jan. 25-29, 1998.

3. L. Lin, A.P. Pisano, R.S. Muller, "Silicon processed microneedles," in Transducers 93', Int. Conf. Solid-State Sensors\&Actuators,pp.237-40, 1993. 4. L. Lin and A.P. Pisano, "Silicon-processed microneedles," in IEEE J. Microelectromech. Sys., vol. 8, no. 1, pp. 78-84, 1999.

5. J. Chen and K.D. Wise, "A multichannel neural probe for selective chemical delivery at the cellular level," in IEEE Solid-State Sensor \& Actuator Workshop, Hilton Head, SC., June 13-16, pp. 256-259, 1994.

6. N.H. Talbot and A.P. Pisano, "Polymolding: two wafer polysilicon micromolding of closed-flow passages for microneedles and microfluidic devices," in Tech. Dig. Solid-State Sensor and Actuator Workshop, Hilton Head, SC., June 8-11, pp. 265-268, 1998.

7. J. Brazzle, I. Papautsky, and A.B. Frazier, "Fluid-coupled metallic microfabricated needle arrays," in Proc. SPIE Micro Fluidic Devices and Systems, Santa Clara, CA, Sep. 21-24, pp. 116-124, 1998.

8. J. Brazzle, S. Mohanty, and A.B. Frazier, "Hollow Metallic Micromachined Needles with Multiple Output Ports", SPIE Micro Fluidic Devices and Systems, Santa Clara, CA, Sept. 20-21, pp. 257-266, 1999.

9. D.V. McAllister, F. Cros, S.P. Davis, L.M. Matta, M.R. Prausnitz, and M.G. Allen, "Three-dimensional hollow microneedle and microtube arrays," Transducers '99, Sendai, Japan, June 7-10, pp. 1098-1101, 1999.

10. I. Papautsky, J. Brazzle, H. Swerdlow, and A.B. Frazier, "A Low Temperature, IC Compatible Process for Fabricating Surface Micromachined Metallic Microchannels", IEEE Journal of Microelectromechanical Systems, (7) 267-73 (1998).

11. I. Papautsky, H. Swerdlow, and A.B. Frazier, "Surface Micromachined IC Compatible Technology for Fabricating Metallic MicroChannels", 10th IEEE International Workshop on Micro Electro Mechanical Systems, Nagoya, Japan, January, 1997, pp. 104-109.

12. J. Brazzle, I. Papautsky, and A.B. Frazier, "Fluid-coupled metallic micromachined needle arrays," in Proc. 20th International Conference of the IEEE Engineering in Medicine and Biology Society (EMBS '98), Hong Kong, Oct. 29 - Nov. 1, pp. 1837-1840, 1998.

13. I. Kadija, V. Chinchankar, V.T. Eckert, E.J. Kudrak, and J. Abys, "Electroplating of thick and ductile palladium: A new electroplating technology," in Proc. of the 77th Annual Conference of the American Electroplaters and Surface Finishers Society, July, 1990.

14. J. Black, Biological performance of materials: Fundamentals of biocompatablity, Marcel Dekker, New York, 1992.

15. S. D. Cramer and D. Schlain, "Electrodeposition of palladium and platinum from aqueous electrolytes," Plating, May, pp. 516-522, 1969.

16. E. J. Kudrak, J. A. Abys, H. K. Straschil, I. Kadija, and J. J. Maisano, "Palladium and palladium alloy plating for the 90's," presented at Connectors '93, East Midlands, England, May 19, 1993.

17. S. Brolin and G. Wettermark, Bioluminescence Anal, VCH Publ., 1992. 18. A. Campbell, Chemiluminescence, VCH Publ., 1989.

20. I.A. Levine, Physical Chemistry $4^{\text {th }}$ ed., McGraw-Hill, 1995, pg. 685.

21. W.H. Safranek, The Properties of Electrodeposited Metals and Alloys, New York: Elsevier, 1974. 